\title{
Preliminary Study of Improving Reservoir Quality of Tight Gas Sands in the Near Wellbore Region by Microwave Heating
}

\author{
Hongcai Wang*, Reza Rezaee, Ali Saeedi
}

Department of Petroleum Engineering, Curtin University, Perth, Australia

Email: hongcaiwang@hotmail.com

\section{Abstract}

The formation damages, such as water blocking and clay swelling, in tight gas reservoir have been recognized as severe problems impairing gas production. To remedy these damages, formation heat treatment (FHT) was taken as one of the effective measures by some researchers. In this paper, the effects of microwave heating on the petrophysical properties of sandstone samples has been investigated. A modified commercial microwave oven was used to heat the core plugs and aluminum tubes were used to accommodate tight sandstone samples in order to confine them and reduce their contact with air. After microwave heating, any alterations in the porosity, permeability, texture, structure, mineralogy, and pore size distribution of tight sandstones were investigated by a series of lab experiments.

By subjecting tight sandstone samples to microwave, the surface temperature of sandstone can be elevated to approximately $400{ }^{\circ} \mathrm{C}$ or more. The intense heat is effective in changing the structure, texture and mineralogy of the sandstone. The shrinkage or decomposition of minerals, which are shown by XRD analysis, and generation of micro-fractures created more spaces in the samples. By employing Automated Permeameter, porosity and permeability are found increased after heating. Nuclear Magnetic Resonance (NMR) and CT numbers of all samples after microwave heating indicate the increase of porosity as well. Moreover, the NMR $\mathrm{T}_{2}$ distribution reveals the smaller pores diminished, so the incremental porosity of short NMR $\mathrm{T}_{2}$ decreased. Micro-fractures generated between grains or in grains due to decomposition of some cement minerals and clay shrinkage, so the amplitude of long NMR $\mathrm{T}_{2}$ increased. The fractures are visible both in X-ray CT images and in Scanning Electronic Microscopy (SEM) images. By comparing with NMR $\mathrm{T}_{2}$ distribution data, it is found that the presence of microfractures accounts for the increased population of pores with $\mathrm{T}_{2}$ larger than $10 \mathrm{~ms}$.

The numerical simulation of microwave heating in the borehole indicates that the microwave heating is effective to raise the temperature of reservoir rock to approximately $900{ }^{\circ} \mathrm{C}$ within 1 day and to remove the water within a distance of $25 \mathrm{~cm}$ from the borehole wall. The efficiency of microwave heating can be further improved by optimizing the downhole microwave device.

\section{Keywords: Tight Gas Sands; Microwave Heating; Pore Size Distribution; Reservoir Quality; Formation Damage; Reservoir Simulation}

\section{Introduction}

As one of the unconventional resources, tight gas is produced from the reservoir with low porosity and permeability. Suitable well stimulation method needs to be performed in such reservoir in order to produce gas in an economical rate (Holditch, 2006). In addition to its poor reservoir quality, the formation damage, which is defined as the impairment of reservoir quality and production, is another crucial problem as well. Due to the intrinsic small pore size in tight gas reservoir and thus the high capillary pressure, the production of tight gas well is susceptible to formation damages such as water blocking and clay swelling. Water blocking refers to the blockage of the pores by the increased water saturation in the near wellbore area due to mud-filtrate invasion. Water blockage will result in reduction of the gas production because of the reduction of relative permeability to gas. Clay swelling is the problem caused by the sensitivity of some clay minerals to fluid salinity. For example, the smectite expands significantly when it is in contact with incompatible fluids in the formation. Clay swelling can 
impede the gas from flowing through the pore throat leading to the reduction of gas flow rate. With the purpose to eliminate such formation damages, some measures have been investigated by several researchers. Their studies provide very valuable information and experiences, although they have more or less disadvantages regarding time efficiency, cost efficiency and environmental friendliness.

However, among them, the thermal methods, especially the microwave heating or high frequency electromagnetic heating, are believed to treat formation damage effectively with the least compromise to the abovementioned disadvantages.

The term, Formation Heat Treatment (FHT) was firstly brought out by (Jamaluddin al., 1995). They applied intense heat, in the form of electrical heater, to the near wellbore area in order to evaporate the moisture phase, to dehydrate, degrade or decompose clay minerals and to create micro-fractures. The thermal shock affects the lattice structure of almost all clay minerals and the degree of changes in the clay lattices depends on the temperature level (Jamaluddin et al., 1998).

The high temperature is the most important factor in the technique of FHT due to the temperature sensitivity of minerals composed of sedimentary rocks. The impact of temperature on clay minerals has been studied by many researchers (Atomic Energy of Canada Limited Research Company, 1990; Grim and Bradley, 1940; Hajpál and Török, 2004; Hoekstra, 1976; Jamaluddin et al., 1998; Li et al., 2006; Mubiayi, 2013; McGill et al., 1995; Sanmiguel et al., 2001; Wu et al., 2011;). Table 1 lists the temperature effects on clay minerals.

Table 1 Effect of Temperature on Clay Minerals (After Carroll, 1970)

\begin{tabular}{|c|c|c|}
\hline Mineral & Temperature, 1 hour & Effect \\
\hline $\begin{array}{l}\text { Kaolinite, well- } \\
\text { crystallized }\end{array}$ & $575-625^{\circ} \mathrm{C}$ & Replacement by amorphous meta-kaolin; no diffraction pattern \\
\hline $\begin{array}{l}\text { Kaolinite, } \\
\text { disordered }\end{array}$ & $550-562^{\circ} \mathrm{C}$ & Similar to kaolinite \\
\hline Dickite & $665-700^{\circ} \mathrm{C}$ & Similar to kaolinite \\
\hline $\begin{array}{l}\text { Mica, well- } \\
\text { crystallized }\end{array}$ & $700^{\circ} \mathrm{C}$ & $\begin{array}{l}\text { Shows gradual loss in weight, but does not break down below } \\
700^{\circ} \mathrm{C} \text {; (001) spacing remain in diffractograms below } 700^{\circ} \mathrm{C} \\
\text { and up to } 1000^{\circ} \mathrm{C}\end{array}$ \\
\hline \multirow{3}{*}{$\begin{array}{l}\text { Illite, and clay } \\
\text { micas }\end{array}$} & $125-250^{\circ} \mathrm{C}$ & Loss of hydroscopic water \\
\hline & $350-550^{\circ} \mathrm{C}$ & Reverts to mica structures \\
\hline & $700^{\circ} \mathrm{C}$ & Similar to mica \\
\hline Glauconite & $58-650^{\circ} \mathrm{C}$ & Loss of interlayer water; reverts to mica structure \\
\hline Biotite & $700^{\circ} \mathrm{C}$ & $\begin{array}{l}\text { Phlogopite is similar to muscovite Biotite shows breakdown } \\
700-1000^{\circ} \mathrm{C}\end{array}$ \\
\hline $\begin{array}{l}\text { Montmorillonite } \\
\text { Group }\end{array}$ & $300^{\circ} \mathrm{C}$ & Original $15 \AA ̊$ spacing disappears; $9 \AA ̊$ spacing develops \\
\hline Chlorite Group & $600-800^{\circ} \mathrm{C}$ & Show gradual weight loss, but no structural change \\
\hline Mg-chlorite & $650^{\circ} \mathrm{C}$ & $14 \AA ̊$ spacing is intensified; (004) at 3.54 not affected \\
\hline Fe-chlorite & $500^{\circ} \mathrm{C}$ & $14 \AA$ spacing less intense and may become broad and diffuse \\
\hline $\begin{array}{l}\text { Mixed-layer } \\
\text { clays }\end{array}$ & $<600^{\circ} \mathrm{C}$ & Varies with kinds of minerals present and amount of each \\
\hline
\end{tabular}

Besides the degradation or decomposition of the clay minerals, the high temperature caused the evaporation of all the free water and partial clay bound water in the pore spaces, which increased the effective permeability to gas. The thermally induced stress in the heated zone may exceeds the yield 
strength of the grains and cements and creates inter-grain and intra-grain fractures (Jamaluddin et al., 1998). The well test indicated that the near wellbore region dehydrated at $382^{\circ} \mathrm{C}$, and the permeability of studied zone increased to $700 \%$ after heating. However, heating the sandstones to temperature of $700{ }^{\circ} \mathrm{C}$ will cause the irreversible changes of clay minerals' structures (Jamaluddin et al., 1999). The study on gas-phase combustion in porous media is another method for FHT. By using this technique, the reservoir can be heated to over $600{ }^{\circ} \mathrm{C}$ leading to the permanent reservoir quality improvement. Li et al. (2006) conducted microwave heating, a novel formation heat treatment technique, on tight sandstone samples and claimed that in microwave active region the energy from the expansion of gas and vaporization and expansion of liquid in the pore spaces can force the trapped fluid to move towards open spaces. The thermal expansive stress can produce a well-interconnected micro-fractures zone that facilitates the flow of moisture vapor and, ultimately gas.

The application of microwave heating in oil and gas industry is not new, it has been utilized in the production of heavy oil due to its efficiency in heating (Abernethy, 1976; Wacker et al., 2011; Carrizales, 2010; Liu and Zhao, 2012). The high frequency electromagnetic wave penetrates the reservoir and heats up reservoir rocks and interstitial fluids within its penetration depth effectively and efficiently. In terms of reservoir with moisture content, the microwave will be almost completely absorbed within a short penetration depth. However, as the water saturation decreased, the penetration depth of microwave can be extended further (Vermeulen and Chute, 1983).

Nevertheless, the application of microwave heating in removing formation damage and enhancing reservoir quality is still challenging. In this paper, the petrophysical and petrographic properties of sandstone samples before and after the treatment of microwave heating in the lab were investigated using multiple experimental techniques. This preliminary study is trying to contribute to the concepts of formation heat treatment by investigating the feasibility of microwave heating in formation heat treatment and providing comprehensive studies of sandstone samples.

\section{Methods and experiments}

Microwave heating is the method to elevate the temperature of the target object by utilizing high frequency electromagnetic wave ranging from $300 \mathrm{MHz}$ to $3 \mathrm{GHz}$. The most common frequency band for microwave processing is $915 \mathrm{MHz}$ and $2450 \mathrm{MHz}$. Two processes are involved in the interaction of microwave and sandstone: polarization, which is the short-range charge displacement, and conduction, which is the long-range charge transport. At low electromagnetic wave frequencies, the ionic conduction losses overwhelm the polarization while the opposite at higher frequencies (Clark and Sutton, 1996). The dielectric losses are decided by both the properties of microwave radiation, such as frequency and power level, and the properties of porous medium, such as the porosity, water saturation and mineralogy. The propagation of electromagnetic wave was described by the Maxwell's equations:

Gauss's law

$\nabla \cdot E=\rho / \epsilon_{0}$

Equation 1

Gauss's law for magnetism

$\nabla \cdot B=0$

Equation 2

Maxwell-Faraday Equation

$\nabla \times E=-\partial B / \partial t$

Equation 3

Ampere's Circuital Law

$\nabla \times B=\mu_{0}\left[J+\epsilon_{0}(\partial E / \partial t)\right]$

Equation 4 
Based on the above equations, the absorbed power per unit volume, $\mathrm{P}$, and penetration depth, $\mathrm{D}$, can be calculated respectively as (Clark and Sutton, 1996):

$P=2 \pi f \epsilon_{0} \epsilon_{e f f}^{\prime \prime}|E|^{2}$

Equation 5

$D=\left[\lambda_{0} / 2 \pi\left(2 \epsilon^{\prime}\right)^{1 / 2}\right]\left[\left(1+\left(\epsilon_{e f f}^{\prime \prime} / \epsilon^{\prime}\right)^{2}\right)^{1 / 2}-1\right]^{-1 / 2}$

Equation 6

The characteristics of microwave propagation are of great importance to the application of microwave heating in FHT. The EM wave can penetrate the formation for a certain distance and heat it up without thermal conduction. From Equation 6, the penetration depth is proportional to the wavelength, which is inversely proportional to frequency. Field measurements of the penetration depth showed that the penetration depth reached $1.33 \mathrm{~m}$ at $2.45 \mathrm{GHz}$ while reached $2.62 \mathrm{~m}$ at $915 \mathrm{MHz}$ (Carrizales, 2010). Wacker et al. (2011) claimed that the microwave heating could penetrate $1.0 \mathrm{~m}$ if the water saturation is $10 \%$ and the penetration depth of inductive heating ranges from $10 \mathrm{~m}$ to $100 \mathrm{~m}$. In addition, the conversion of microwave to heat can be completed in a short time, so the heating time is minimal. In this paper, a modified commercial microwave oven has been used to implement heating experiments. Its operating frequency is $2450 \mathrm{MHz}$, and there are different levels of heating power with the maximum level of $1050 \mathrm{~W}$. The oven was equipped with high temperature insulation in order to suit the requirements of high temperature heating. The samples were tightly wrapped with aluminum tube, which is transparent to microwave, in order to provide the confining pressure and reduce samples' contact with air. The temperature was measured with an infrared thermometer with an accuracy of $1 \%$. The properties of sandstone core plugs are listed in Table 2.

Table 2 Properties of sandstone core plugs

\begin{tabular}{|c|c|c|c|c|c|c|}
\hline Sample ID & Field & Well Name & $\begin{array}{l}\text { Length } \\
(\mathrm{cm})\end{array}$ & $\begin{array}{l}\text { Porosity@500 } \\
\text { psi }\end{array}$ & $\begin{array}{l}\text { Permeability@500p } \\
\text { si (mD) }\end{array}$ & Lithology \\
\hline WR-01 & $\begin{array}{l}\text { Whicher } \\
\text { Range }\end{array}$ & Whicher Range 4 & 4.851 & $3.745 \%$ & 0.028 & Sandstone \\
\hline WR-11 & $\begin{array}{l}\text { Whicher } \\
\text { Range }\end{array}$ & Whicher Range 4 & 4.980 & $10.844 \%$ & 0.211 & Sandstone \\
\hline WR-14 & $\begin{array}{l}\text { Whicher } \\
\text { Range }\end{array}$ & Whicher Range 4 & 4.487 & $3.58 \%$ & 0.054 & Sandstone \\
\hline ERREG-2 & Erregulla & Erregulla 2 & 4.933 & $3.362 \%$ & 0.013 & Sandstone \\
\hline
\end{tabular}

The following experimental procedures were adopted for all sandstone samples before and after microwave heating:

- Small pieces of samples were separated from the core plugs before and after any experiment and were used for the Scanning Electronic Microscopy (SEM) imaging and X-ray Diffraction (XRD) analysis.

- Dry the sample in the lab oven at lower temperature $\left(50{ }^{\circ} \mathrm{C}\right)$ for 24 hours and then measure their porosity and permeability with an automated permeameter before and after MW heat treatment.

- Subject the whole core plugs to X-ray CT scanning with a medical X-ray CT.

- Saturate the sample with 20,000-ppm brine in a high-pressure (2000-psi) saturator and then conduct NMR test for original and heated samples.

The Automated Permeameter uses helium/nitrogen to measure the porosity and permeability under overburden pressure. It accommodates core plug with 1.5-inch diameter. The inputs include dimensional information and the outputs are the porosity, pore volume, permeability and Klinkenberg-corrected permeability.

The Nuclear Magnetic Resonance (NMR) test was completed on a 2MHz NMR rock core analyzer. 
Samples need to be fully saturated before measuring the pore size distribution and porosity. Besides, information such as irreducible water saturation and clay bound water saturation can be obtained from this equipment by preparing samples accordingly.

The internal structure and texture can be reconstructed after conducting medical X-ray Computational Tomography (CT) scanning. The advantage of this method is that it can provide the overall information of whole samples in no time. With the assistance of image processing software, the presence of microfractures can be easily identified. Furthermore, the density/porosity change of core plugs along the sample length was proved to correlate with the CT numbers, which can be calculated in the software conveniently. Such comparable information gives us a good understanding on the quantitative and qualitative alteration of sandstone samples. Nevertheless, the disadvantage is that the resolution of Xray CT scanning is not sufficiently high to investigate details of the microstructures, so the Scanning Electron Microscope (SEM) was employed to obtain the information of minerals and microstructures of rock samples as a complement of X-ray CT scanning. A very small piece of sample is needed for SEM, and it is important to note that the surface of the sample should not be prepared by cutting but by breaking. A $4 \mathrm{~nm}$ platinum coating was carefully applied on the sample and was subject to SEM after vacuuming for one week. All our samples were prepared in the same way and same equipment, and the imaging settings were kept the same in order to minimize the error caused by equipment and procedures.

\section{The Effect of microwave heating on the reservoir quality}

The thermal method is effective in improving both porosity and permeability. The traditional FHT indicates the porosity and permeability increased many folds in the near well-bore area and brought great economical potential. Instead of heating with an electrical heater or gas combustion, we used the microwave as heat source. As shown in Figure 1, the reservoir quality of all core plugs studied were enhanced. The porosity increased by $15.6 \%, 50.8 \%, 6.8 \%$ and $14.2 \%$ respectively for sample ERREG-2, WR-01, WR-11 and WR-14, while the permeability had greater growth by $238.5 \%, 728.6 \%, 315.2 \%$ and $464.0 \%$ respectively. We found that the slight change in porosity leads to a large improvement in permeability, and the configuration of pore sizes is the factor that needs to be closely investigated.

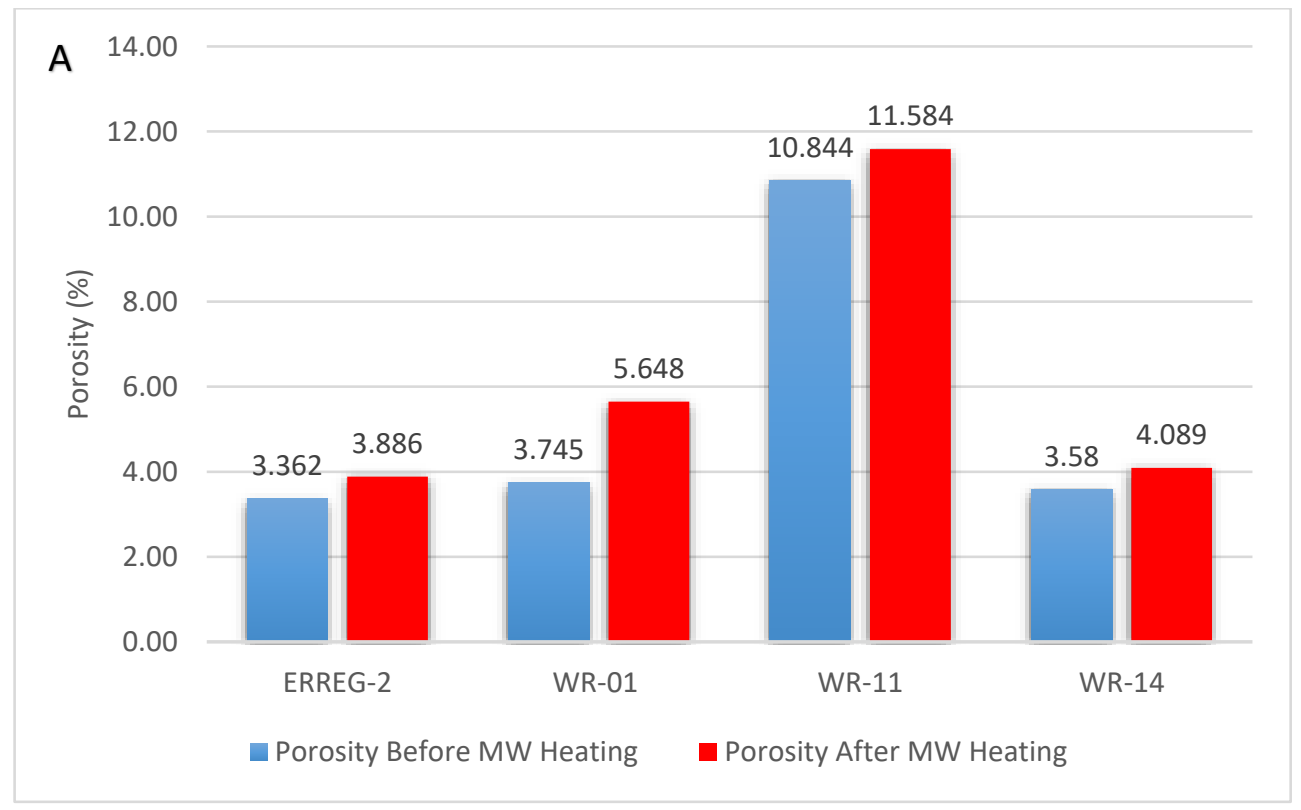




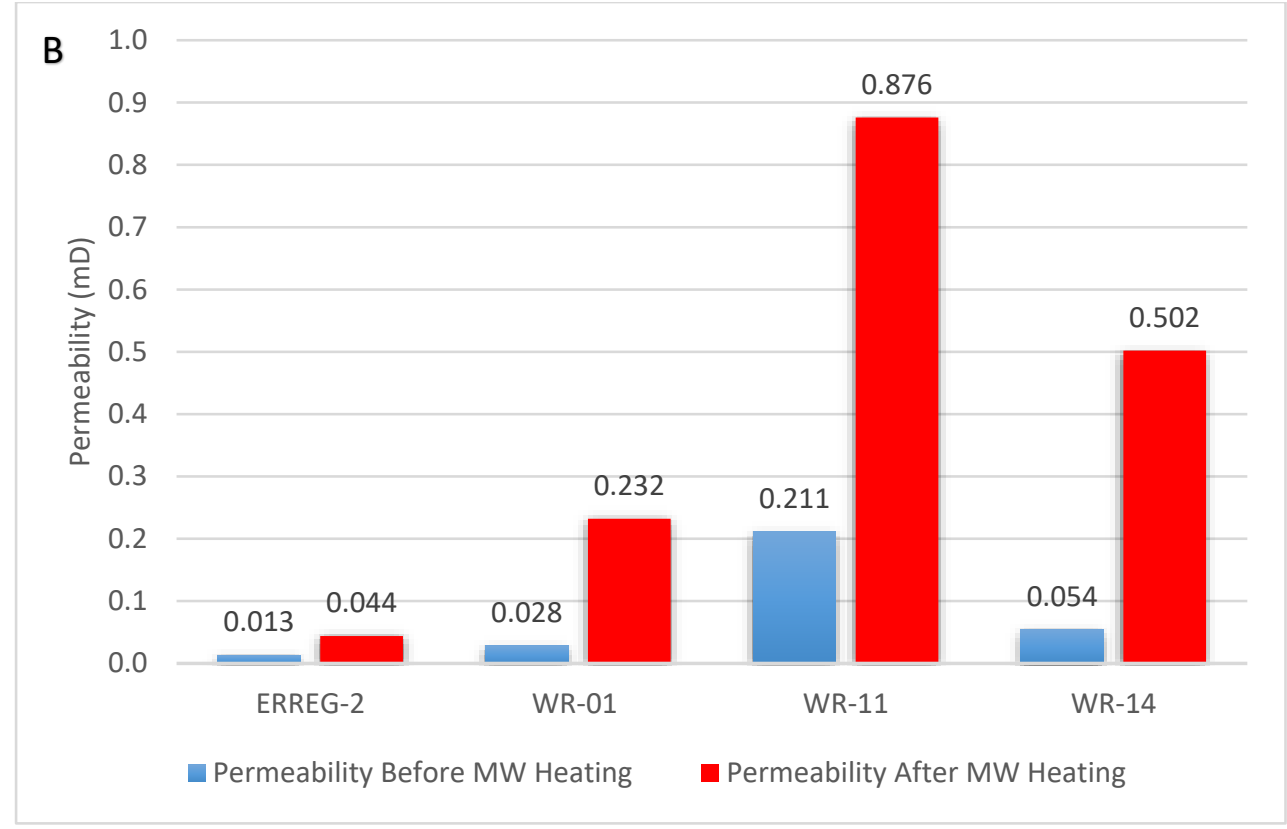

Figure 1 Comparison of porosity $(\mathrm{A})$ and permeability $(\mathrm{B})$ before and after microwave heating

NMR transverse relaxation $\left(\mathrm{T}_{2}\right)$ is induced by the interaction of protons in the magnetic field. For core plugs saturated with brine, the bulk fluid relaxation, surface relaxation and diffusion are three contribution mechanisms (Coates et al., 1999). After being fully saturated with water, the $\mathrm{T}_{2}$ value of a single pore in the water-wet rock samples is proportional to the size of the pore: the longer $\mathrm{T}_{2}$ indicates larger pores while the shorter T2 indicates smaller pores. In the actual sandstone sample, pores of different sizes are mixed together and these signals are measured all together. By performing echo-fit, the $\mathrm{T}_{2}$ distribution can be determined. The amplitude of $\mathrm{T}_{2}$ distribution curve yields porosity information as well, since the area under the $\mathrm{T}_{2}$ profile is proportional to the number of hydrogen protons in the pore fluids (Coates et al., 1999). (Coates, et al., 1999). To simplify the demonstration in this paper, the pore sizes were divided into three groups according to their NMR $\mathrm{T}_{2}$ values, and they are defined as below: smaller pores (less than $0.1 \mathrm{~ms})$, medium-size pores $(0.1$ to $10 \mathrm{~ms})$ and larger pores (greater than $10 \mathrm{~ms}$ ). The encouraging findings are shown in Figure 2. As the tight sandstone samples have been fully saturated for the NMR experiments, the area under the $T_{2}$ curve from $1 \mathrm{~ms}$ to $100 \mathrm{~ms}$ demonstrated an obvious increased porosity of heated samples. In sample ERREG-2, the population of medium-size pores and larger pore had a slight growth after heating with microwave, however, the amplitude was found decreased at around $0.1 \mathrm{~ms}$ and between $1 \mathrm{~ms}$ and $10 \mathrm{~ms}$. Similarly, the population of pores, with $\mathrm{T}_{2}$ ranging from 0.1 to $0.3 \mathrm{~ms}$, decreased in sample WR-1, while the population of medium-size and larger pores increased, with $\mathrm{T}_{2}$ ranging from 0.6 to $30 \mathrm{~ms}$.

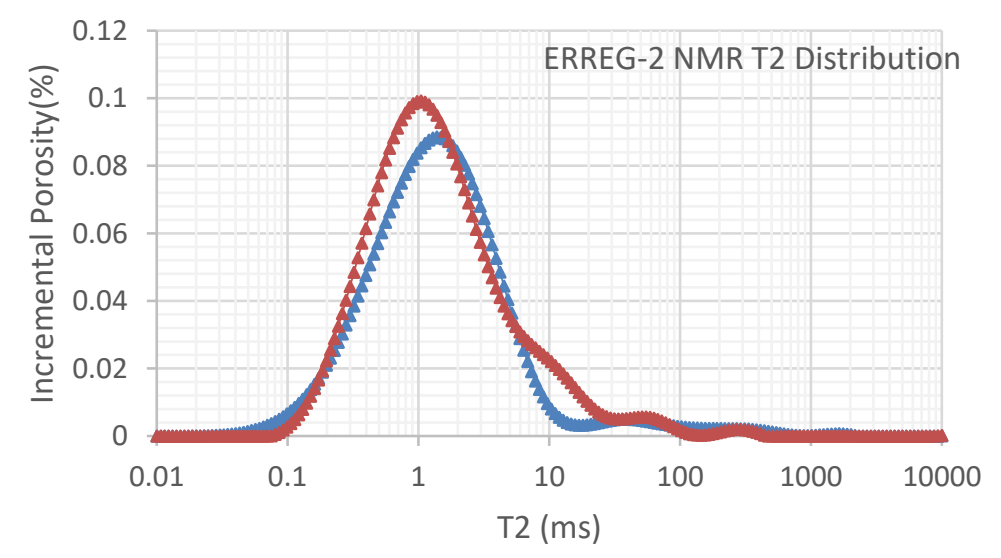



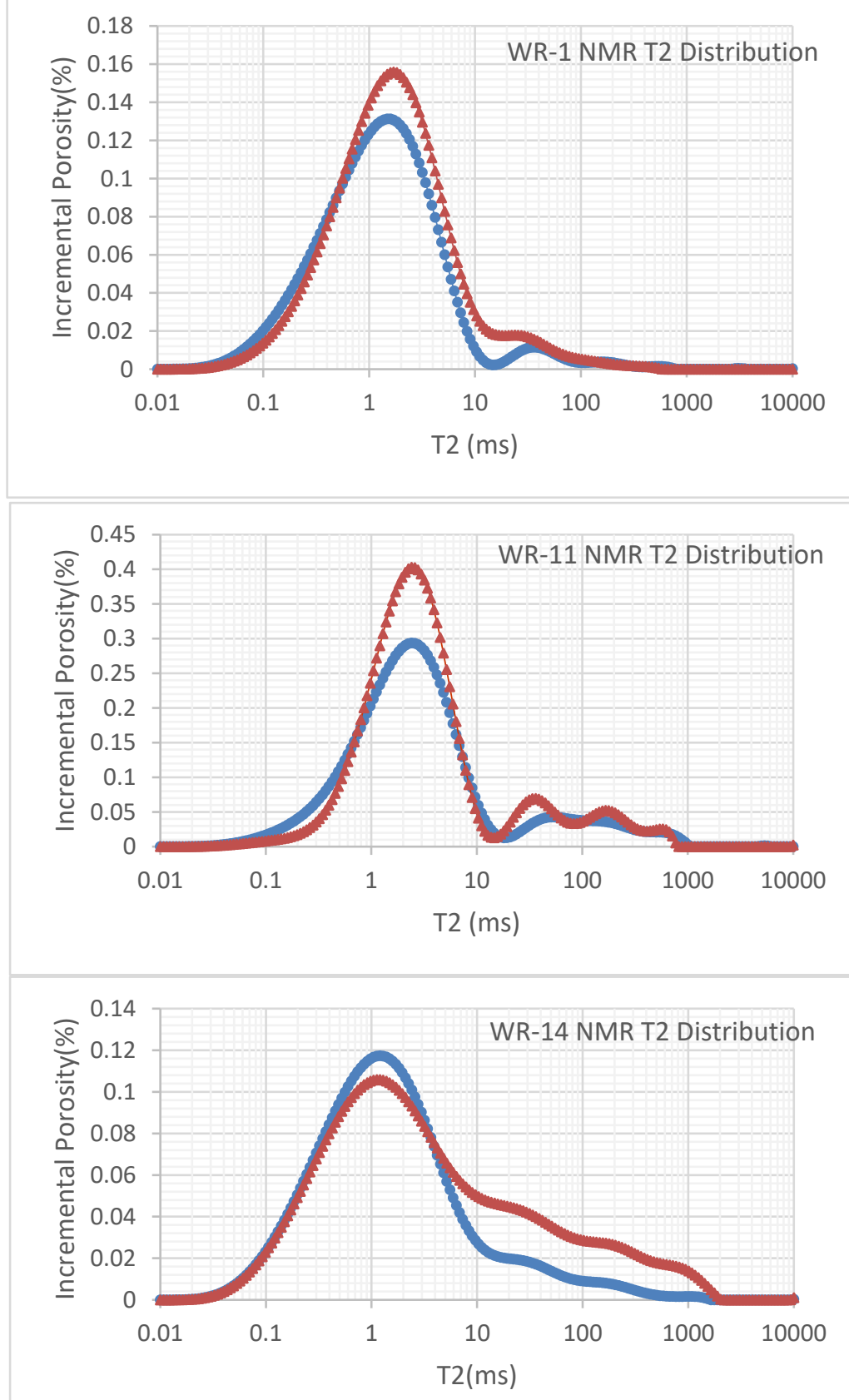

$\longrightarrow$ Before MW Heating $\quad$ —After MW Heating

Figure 2 Comparisons of NMR Experiments Results of 4 Tight Sandstone Samples. (The X-axis represents the transverse relaxation time $\left(\mathrm{T}_{2}\right)$, the longer $\mathrm{T}_{2}$ the larger pore size; the $\mathrm{Y}$-axis is the incremental porosity, which represents the percentage of the specific pore size (or T2), and its sum (from T2=0.01ms to 10000ms) should be 1. After microwave heating, there is slight drop in smaller pores $(<0.1 \mathrm{~ms})$, obvious increase in medium-size pores (0.1-10ms) and slight increase in larger pores of sample ERREG-2, WR-1 and WR-11. In WR-14, there is slight decrease in medium-size pores but great increase in larger pores.

The alteration of pore size distributions is due to the microwave heating changed the grains mechanically or chemically, and thus changed pore space between these grains. The connection or expansion of smaller pore spaces created larger pores. In other words, the decrease of smaller pores contributed to the growth of the amount of larger pores. This can be clearly demonstrated in sample WR-11. In sample WR-14, the change of pore size distribution is different from the other three samples: the population of medium-size pores decreased but the larger pores increased more drastically. In fact, 
the temperature of the inner part of sample WR-14 was measured roughly and it reached higher than $700^{\circ} \mathrm{C}$. In addition to the greater heterogeneity, large fractures have been created along beddings in sample WR-14 (Figure 6).

\section{Porosity Correlation}

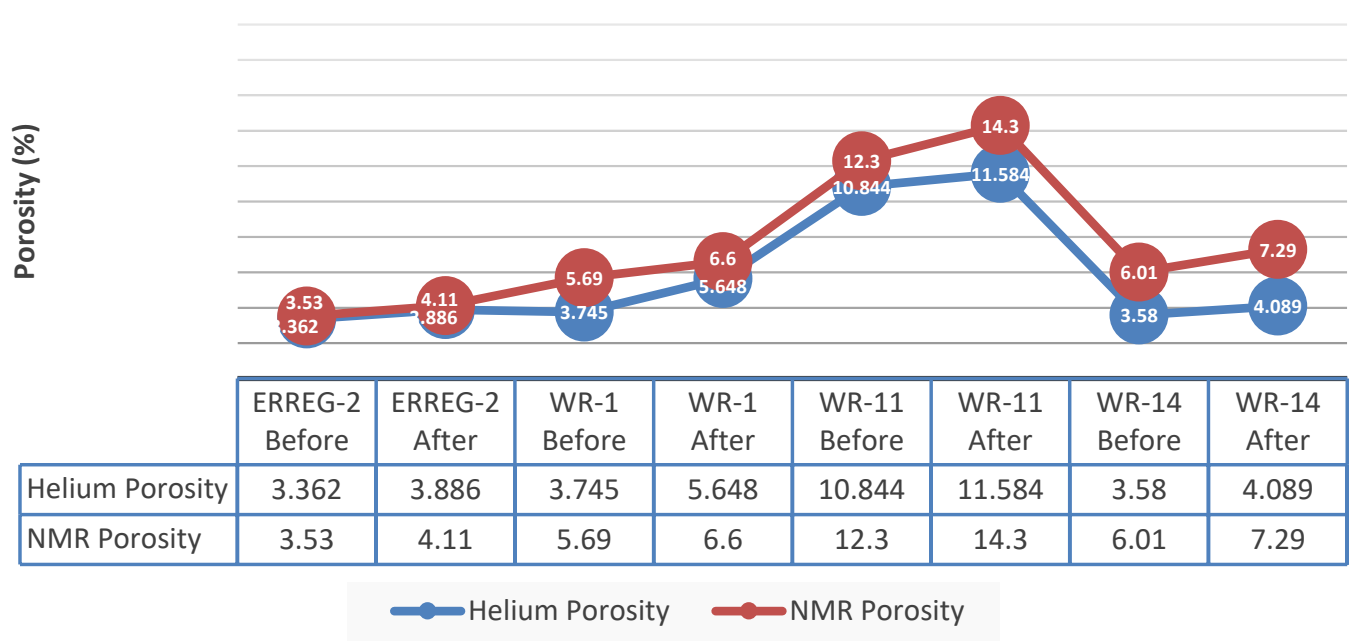

Figure 3 Porosity Correlation of Helium Porosity and NMR Porosity Before and After Microwave Heating.

The decomposition of clay minerals with micro-pores might lead to release of more pore spaces held by those micro-pores. Hajpál and Török (2004) indicated that the micro-fractures will develop at grain boundaries when temperature is over $600^{\circ} \mathrm{C}$ and the higher temperature at $750^{\circ} \mathrm{C}$, is necessary for fractures within crystals.

The helium porosity and NMR porosity for each sample were correlated to calibrate the NMR test results. As indicated in Figure 3, a similar trend of porosity change has been found in all the involved samples. For example, the slope between "ERREG-2 Before" and "ERREG-2 After" of helium porosity is the same as the slope of NMR porosity.

\section{The effects of microwave heating on tight sandstones}

The presence of clay minerals affects the reservoir quality significantly. The typical occurrences of clay minerals in sandstone are pore lining, pore bridging and pore filling. These minerals can impair the permeability by: (1) migrating to the pore throat and block the flow channel; (2) swelling when contact with incompatible fluids and block the pores.

Micro-fractures, which are induced by differential stresses, are small cracks with high aspect ratio (Anders et al., 2014). In this paper, the micro-fractures we studied are generated due to the thermal stress or differential thermal expansion. Grains in the tight sandstones have different thermos-elastic moduli and thermal conductivities. Consequently, when exposed to high temperature, these grains have different thermal expansions generate intergranular micro-fractures (Kranz, 1983).

Non-destructive imaging techniques are applied to the tight sandstone samples in this paper. The medical X-ray CT scanning images provide the three dimensional information of tight sandstone samples as well as the CT numbers. CT numbers are calculated to illustrate the variation of porosity along the length of samples after heating, which provide a straightforward and reliable method to compare samples' property. The SEM focuses on the small pieces of samples and provides highresolution images. It is good at investigating the changes in clay minerals, micro-fractures and micropores, so we utilized it to study the dehydration of clays, thermal-induced fractures and any changes in microstructure. 


\subsection{Medical x-ray CT scanning}

The X-ray CT scanning is a non-destructive method to study the internal structure of sandstones. The core plugs are intact and suitable for other measurements. CT attenuation data are presented in Hounsfield Unit $(H U)$, which is given by the CT Number of air at -1000 and of water at $0 \mathrm{HU}$ (Wellington and Vinegar, 1987). The CT Numbers are important parameters yielded from X-ray CT scanning, which are directly correlated to grain density (Perez, 1993). For sandstone CT scanning experiments, a change of $\pm 1 H U$ is equivalent to a fractional density change of $\pm 0.5 \times 10^{-3}(\Delta \rho / \rho)$ (Wellington and Vinegar, 1987).

Sandstones are composed of grains, which are mainly quartz and feldspar; cements, which are mainly clays; and pores filled with air when running CT scanning. In CT images, pores are identified by low CT numbers and the minerals are identified by high CT numbers (Yao et al., 2009). In other words, the lower CT numbers the higher porosity.

As shown in Figure 4, in general, the CT Numbers of four samples, ERREG-2, WR-1, WR-11 and WR14, dropped after heating but the dropping degrees are different from sample to sample. In most of the samples, even the variations in the sample are not homogeneous in different part.

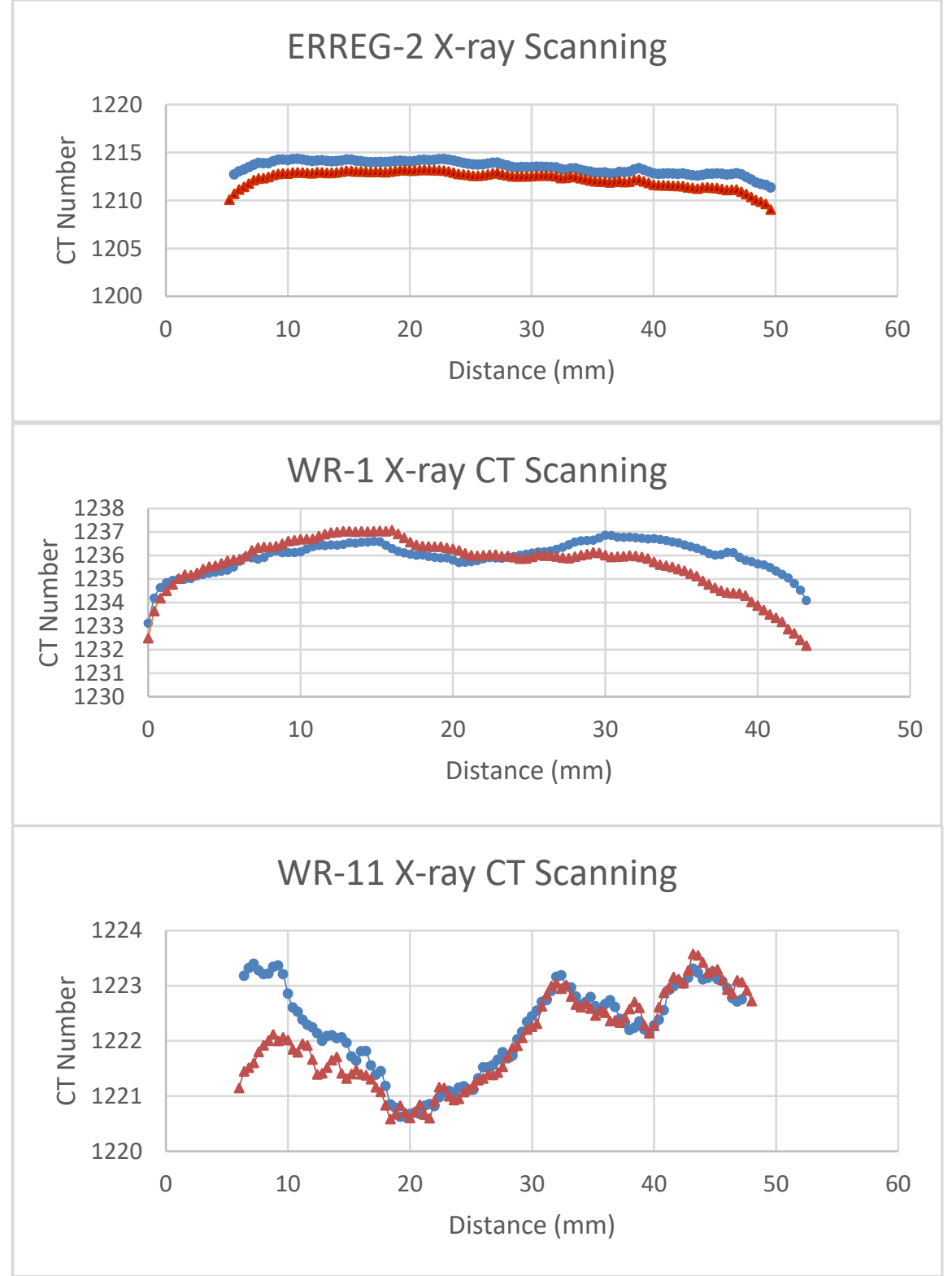




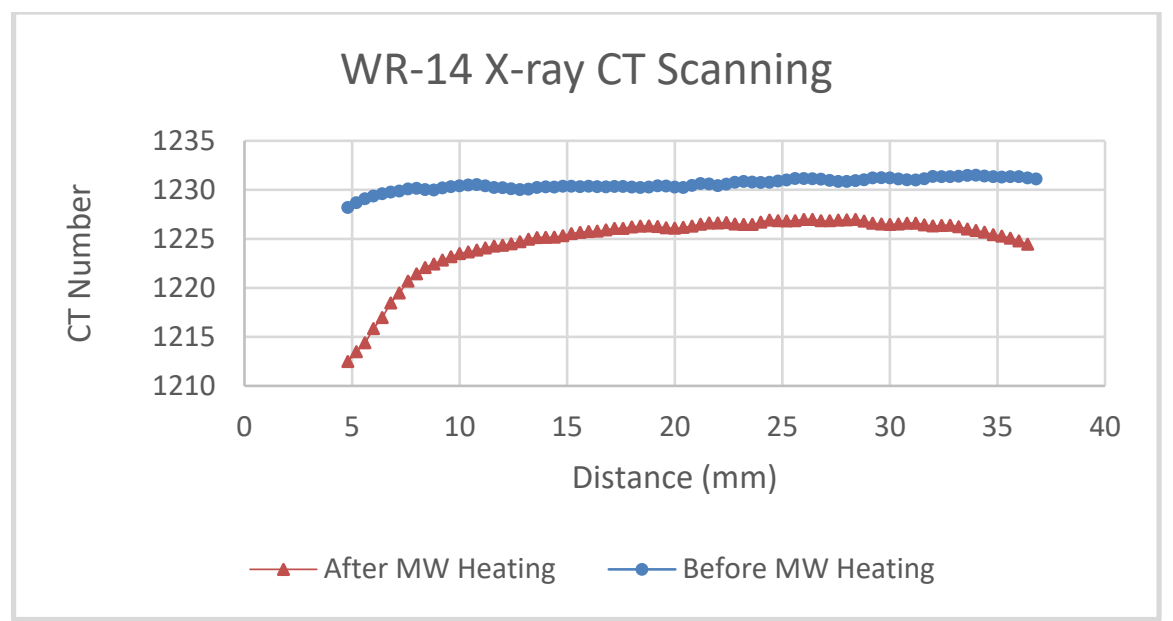

Figure 4 Comparisons of CT Numbers Before and After Heating (High CT number represents low porosity, while low CT number represents high porosity. After microwave heating, the CT numbers, which are calculated in the same software, decreased for the samples).

It is obvious that the CT numbers reduced (porosity increased) after microwave heating. In detail, for sample ERREG-2, the porosity increases are homogeneous through the sample, because the X-ray CT profiles are parallel with each other and the CT number decreased. Similarly, the overall porosity improvement is observed in sample WR-14, and there is greater porosity improvement (CT number decreased) at one end of the core plug. By investigating the X-ray CT images of WR-14 (Figure 6), we found that the visible fracture from one end of the sample and it extended to the internal along the length direction. In the direction of diameter, the maximum fracture length almost equals to the diameter, which accounts for the great enhancement of porosity. As the heating method is microwave radiation, according to its principles, the heating effect relies on the electric field distribution, which is not even in the target object. In addition, the dielectric properties of minerals are different, so their temperatures vary after exposing to the microwave. Moreover, these minerals have various thermal expansion properties, so there are differences in their response to microwave heating. These combined factors lead to the presence of the fractures in the heated sample. Another example is in sample WR-1 (Figure 5), which shows the fractures at one end of the sample. However, the dimensions of the fracture are much smaller than in WR-14. Besides the above three factors, we also should take the consideration of the structure of the sample: compared with WR-1, WR-14 is more laminar (more heterogeneous). This laminar structure facilitates the generation and development of fractures during microwave heating. 


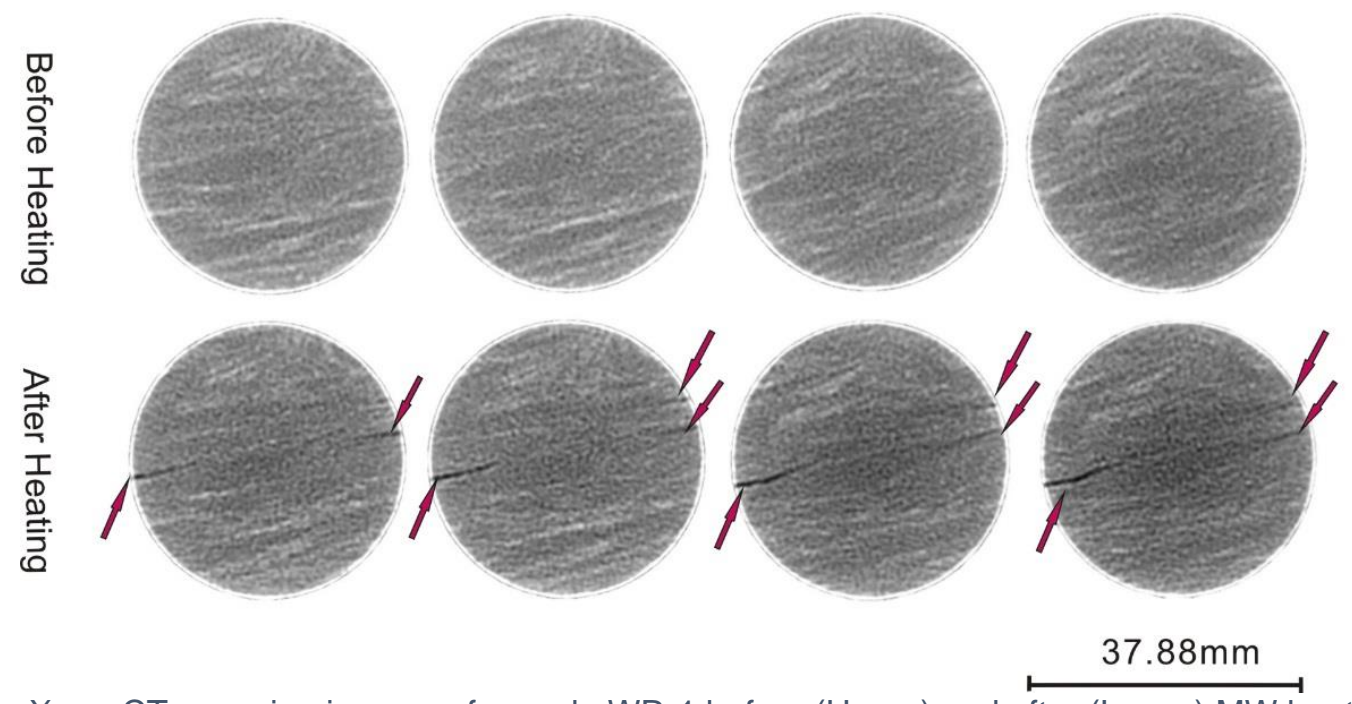

Figure 5 The X-ray CT scanning images of sample WR-1 before (Upper) and after (Lower) MW heating. These cross section images are taken from the same position of the core plug before and after heating to demonstrate the presence of fractures due to microwave heating. The red arrow indicates the position of fractures.
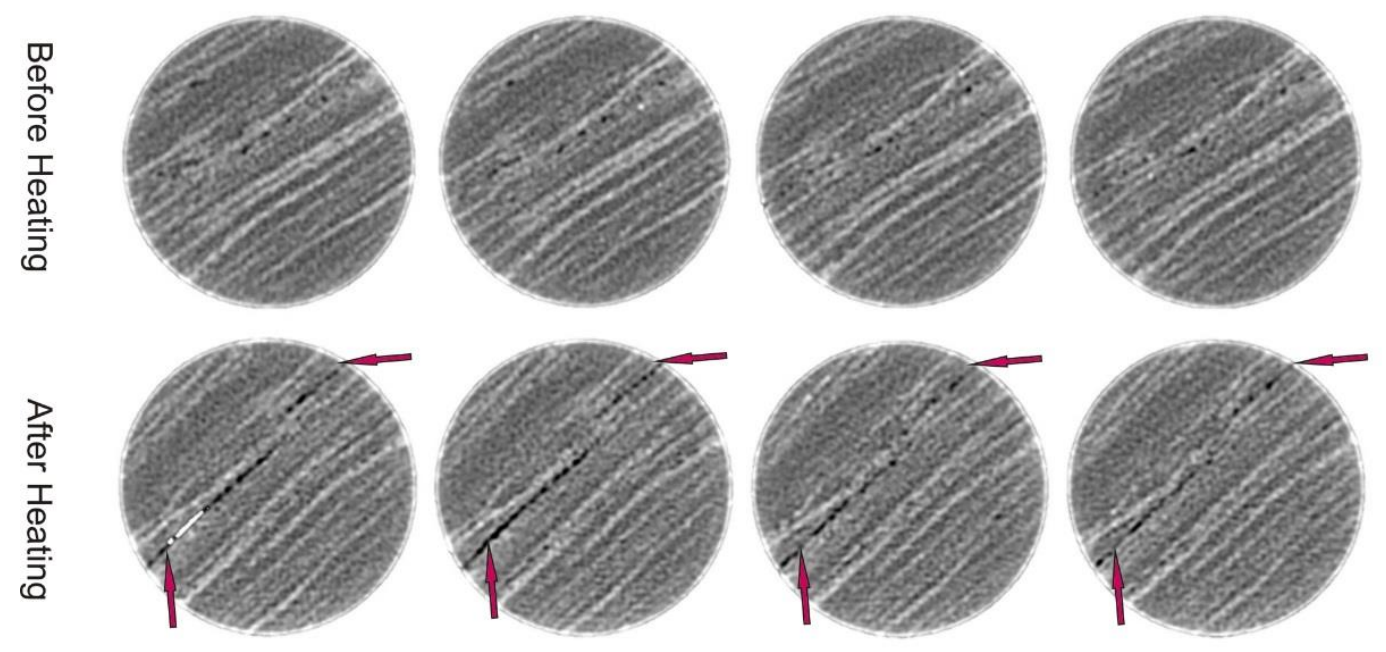

$37.82 \mathrm{~mm}$

Figure 6 The X-ray CT scanning images of sample WR-14 before (Upper) and after (Lower) MW Heating These cross section images are taken from the same position of the core plug before and after heating to demonstrate the presence of fractures due to microwave heating. The red arrow indicates the position of fractures.

\subsection{Scanning electronic microscope}

The microstructures have been investigated with SEM technique at different magnifications before heating and after heating. The morphology of the fractures and mineralogy are closely examined to reveal the effect of microwave heating on tight sandstones. The fractures in the sandstone are influenced by minerals, textures and microstructures of the sample, and the distribution of internal micro defect and nucleation. Based on different fracture morphology characteristics, the fracture mechanisms will include the inter-granular fracture, trans-granular fracture, clay cement fracture and the coupled inter granulartrans-granular fracture (Xie et al., 1989; Zuo et al., 2007).

The experiments on isolated kaolinite revealed that the structure of kaolinite will be destroyed at $550{ }^{\circ} \mathrm{C}$ 
(Jamaluddin et al., 1998). In addition, Carroll (1970) reported that the well-crystallized kaolinite would be replaced by amorphous meta-kaolinite when temperature reaches $575-625^{\circ} \mathrm{C}$ (Jamaluddin et al., 1998).

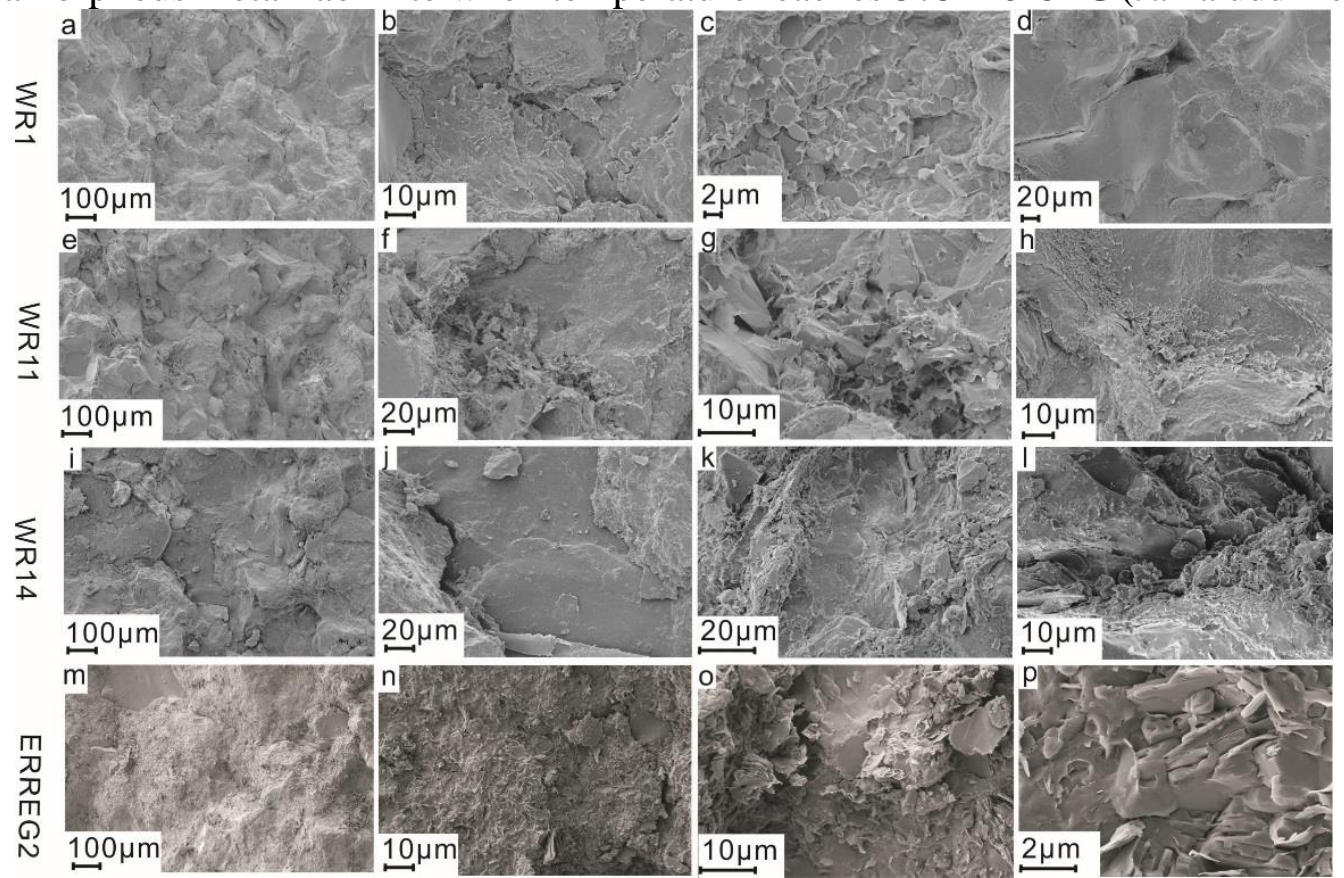

Figure 7 SEM images of samples before microwave heating. Grains are attached with each other closely and fractures are hardly to be spotted in these images.

Before microwave heating, tight sandstone samples, WR-1, WR-11, WR-14, and ERREG-2 shown in Figure 7, are highly cemented, and no fractures are observed in these samples with magnification of 100. In terms of reservoir quality, all four samples are poor. Quartz composed most of the samples, while some clay minerals can be found in the images (Figure 7-g, smectite, Figure 7-i, illite, and Figure 7-p, kaolinite). After heating, fractures in all samples are widely observed in both low and high magnifications. In Figure 8, the fractures are visible at a relatively low magnification. Besides, the numbers and widths of the fractures are increased compared with the according samples before being heated. Fractures are observed along the boundaries of grains and in the clay minerals. Combined with NMR T2 distribution data (Figure 2), the presence of fractures accounted for the increased population of T2 larger than $10 \mathrm{~ms}$. 


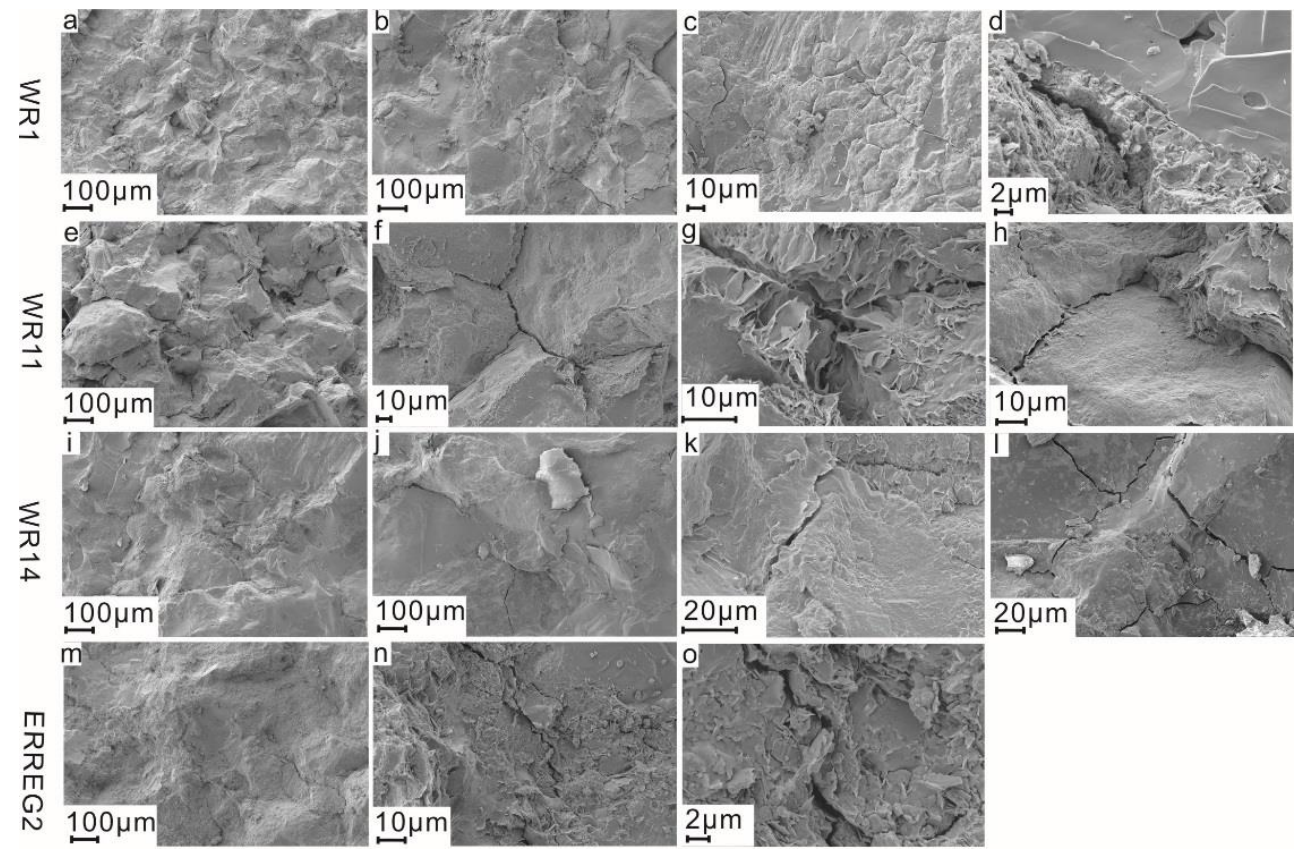

Figure 8 SEM images of samples after microwave heating. Fractures can be widely found in samples after heating, $f$ and $\mathrm{h}$ indicate the intergranular fractures, $\mathrm{g}$ indicates the fractures caused by clay shrinkage and $\mathrm{I}$ indicates the intergranular fractures.

In terms of the mechanisms of fractures creation, there are two main mechanisms:

1) Extra pore or micro-fractures are created among clay minerals because of their volume shrinkage induced by clay dehydration or degradation (Figure 8-g and Figure 8-o). (Li, Meng, Tang, \& Inst, 2006)

2) Intergranular fractures generated between grains due to the differential thermal expansion and decomposition of cement between the grains (Figure 8-f and Figure 8-h). Intragranular fractures formed in grains because of the thermal expansion and surrounding thermal stress (Figure 8-1). 


\subsection{X-ray Diffraction (XRD) analysis}

The composition changes of tight sandstone samples have been studied with XRD analysis. As the most abundant minerals in tight sandstone, quartz did not show changes in peak shape in Figure 9 and Figure 10 , the slight peak shift to the higher degree might indicate the phase change of quartz. The improvement of reservoir quality are more likely to be linked with the changes of the other minerals.

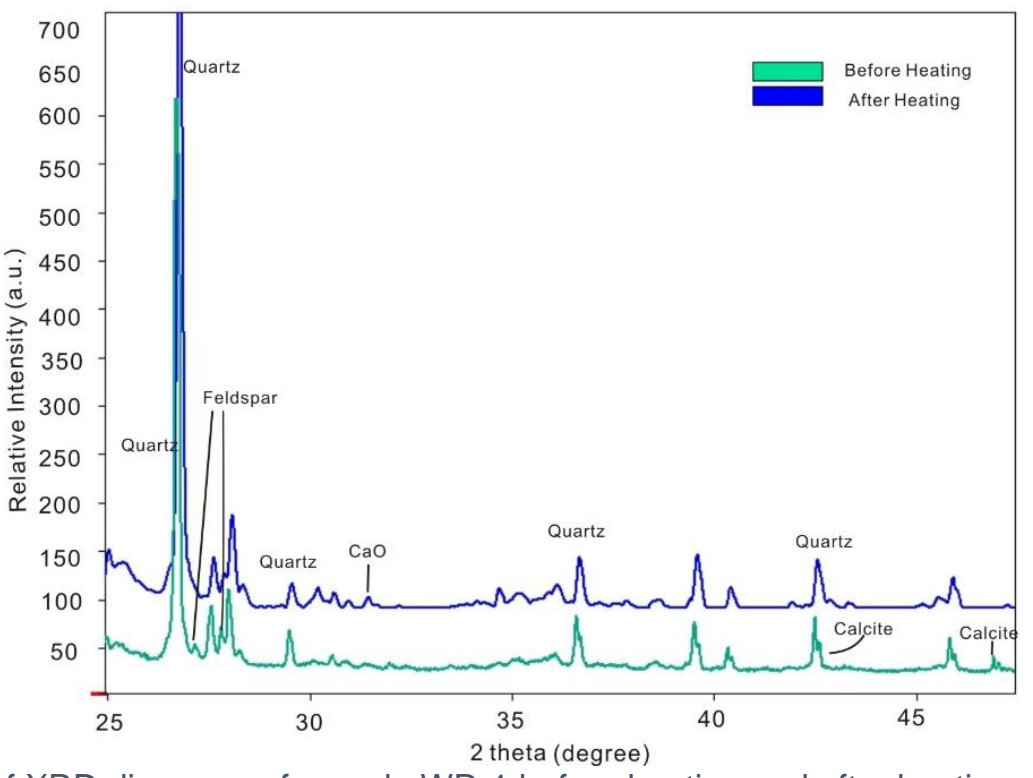

Figure 9 Comparison of XRD diagrams of sample WR-1 before heating and after heating. Peaks of quartz shift to the right slightly after heating. Calcite presented in the sample before heating while it disappeared after heating. Instead, $\mathrm{CaO}$ appeared after heating, which could be the product of Calcite decomposition.

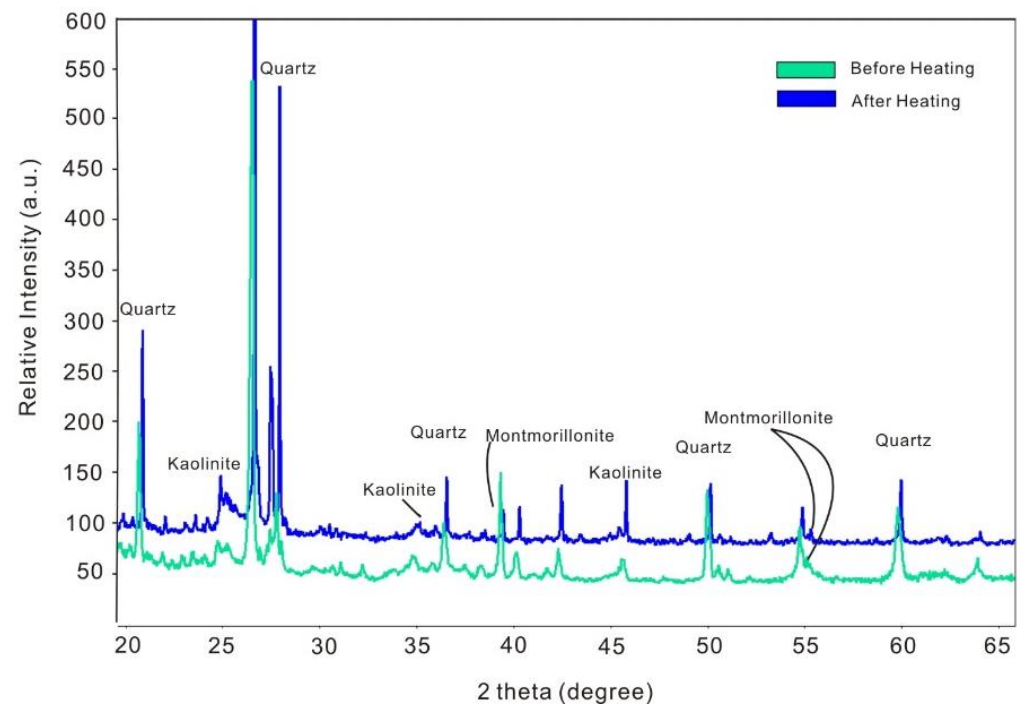

Figure 10 Comparison of XRD diagrams of sample WR-14 before heating and after heating. After heating, peaks of quartz and kaolinite shift to the right slightly after heating and the relative intensity of montmorillonite reduced.

As indicated in Figure 9, the microwave heating treatment decomposed calcite and produced $\mathrm{CaO}$. Since calcite may play a role of cementing grains in sandstone, the reservoir quality becomes poor when it presents in the sample. By heating the reservoir rock, the calcite cement can be decomposed, so the porosity and permeability of sandstones can be improved. At the same time, the peaks of feldspar, which is another common cementing mineral, disappeared after heating. The disappearance of feldspar contributes to the reservoir quality enhancement as well. Finally, structure changes in montmorillonite group minerals, which can swell when in contact with fresh water, are shown by the decreased relative intensity in Figure 10. 


\section{Numerical Simulation}

Reservoir simulation has been conducted to investigate the water invasion and formation heat treatment with microwave heating. To simulate the water invasion, the well was injected water for 3 days with daily injection rate of $5 \mathrm{~m}^{3}$. After that, the well was heated with microwave for 1 day. Microwave heating in four different directions $(-\mathrm{X},+\mathrm{X},-\mathrm{Y},+\mathrm{Y})$ in the wellbore was simulated simultaneously. The dimensions for the simulated area are $3.5 \mathrm{~m}$ (length) $* 3.5 \mathrm{~m}$ (width) $* 10 \mathrm{~m}$ (thickness). The reservoir properties are listed in Table 3.

Table 3 Parameters used in the numerical simulation.

\begin{tabular}{ll}
\hline Parameter & Value \\
\hline Reservoir Temperature $\left({ }^{\circ} \mathrm{C}\right)$ & 139 \\
Reservoir Porosity $(1)$ & 0.1 \\
Reservoir Permeability $(\mathrm{mD})$ & 0.1 \\
Initial Water Saturation $(1)$ & 0.3 \\
Critical Water Saturation $(1)$ & 0.6 \\
Reservoir Depth $(\mathrm{m})$ & 4000 \\
Thermal Gradient $\left({ }^{\circ} \mathrm{C} / 100 \mathrm{~m}\right)$ & 2.8 \\
Surface Temperature $\left({ }^{\circ} \mathrm{C}\right)$ & 27 \\
Thermal Conductivity $\left(\mathrm{J} /\left(\mathrm{m} \cdot\right.\right.$ day $\left.\left.\cdot{ }^{\circ} \mathrm{C}\right)\right)$ & $1.73 \times 10^{5}$ \\
Heat Capacity $\left(\mathrm{J} /\left(\mathrm{cm}{ }^{3} \cdot{ }^{\circ} \mathrm{C}\right)\right)$ & 2.385 \\
Microwave Frequency $(\mathrm{MHz})$ & 2450 \\
Operation Power $(\mathrm{Watt})$ & 10000 \\
\hline
\end{tabular}

After injecting water for 3 days, the water saturation in the near wellbore area increased from 0.3 to around 0.8 and the invasion depth reached up to $0.7 \mathrm{~m}$ (Figure 12, a). This agrees well with the approximate depth of invasion in an actual tight gas well, which is around 0.43 to 0.90 meters (Miesch and Albright, 1967; Rider, 1986) (Table 4).

Table 4 Depth of invasion (distance from borehole wall) versus porosity (after Miesch and Albright (1967) and Rider (1986))

\begin{tabular}{lllll}
\hline Hole Size (in) & 17.5 & 12.25 & 8.5 & $\begin{array}{l}\text { Ratio invasion } \\
\text { diameter: hole } \\
\text { diameter }\end{array}$ \\
\hline Porosity (\%) & \multicolumn{4}{c}{ Depth of invasion $(\mathrm{cm})$} \\
\hline $1-8$ & 200.0 & 140.0 & 97.0 & 10 \\
$8-20$ & 90.0 & 62.0 & 43.0 & 5 \\
$20-30$ & 22.5 & 15.5 & 11.0 & 2 \\
$30+$ & 3.0 & 2.0 & 1.7 & $<2$ \\
\hline
\end{tabular}

After microwave heating for 1 day, the maximum temperature in the near wellbore region reached to about $900^{\circ} \mathrm{C}$ and the depth influenced by the heat reached around $0.4 \mathrm{~m}$. In this simulation, we focused on the penetration depth of microwave heating, temperature distribution and its effect on water saturation. The effects of microwave heating on relative permeability and gas production have been discussed in another paper (Wang, Rezaee and Saeedi, 2015). The water saturation in the near wellbore region was reduced significantly. As shown in Figure 12-b, the water phase was completely removed within about 25 centimetres from the wellbore wall. Compared with Figure 12-a, the moisture content in the further distance away from the wellbore dropped to a relative low level but not zero. However, it is convinced that by increasing microwave heating power and heating time, the penetration depth of treatment can be enlarged and the water saturation can be further reduced. 


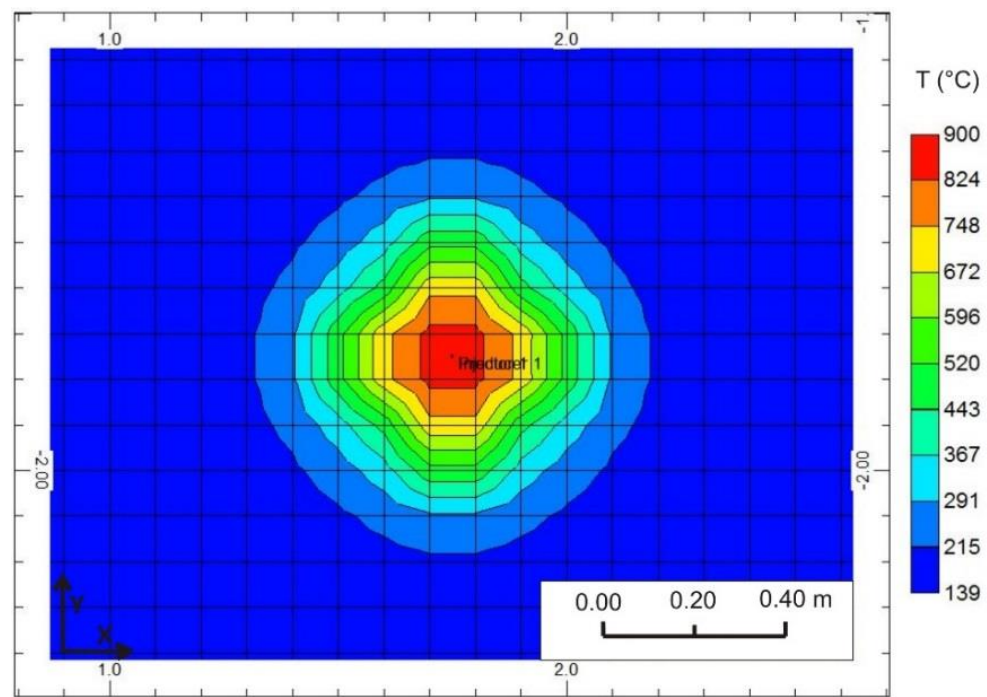

Figure 11 Temperature distributions around the wellbore after 1-day microwave heating. The maximum temperature reached is approximately $900^{\circ} \mathrm{C}$, while the area, which is about $40 \mathrm{~cm}$ from the wellbore, reached approximately $250^{\circ} \mathrm{C}$.

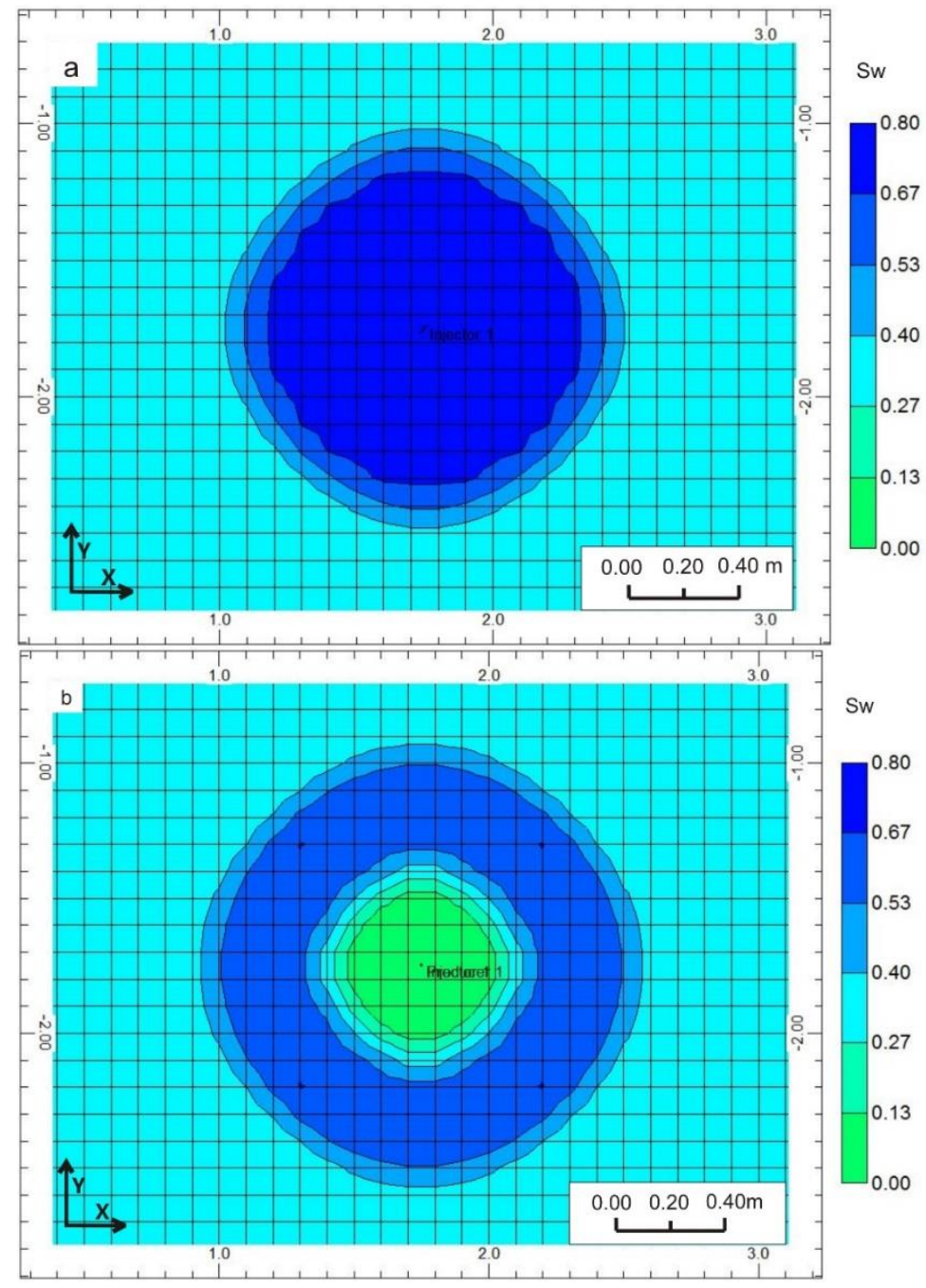

Figure 12 Water saturation distributions around the wellbore: (a) after 3-day water injection, the water saturation in the near wellbore region increased to 0.8 ; (b) after 1-day microwave heating, the water within $25 \mathrm{~cm}$ from the borehole wall has been completely removed. 


\section{Discussions}

This paper studied the feasibility of formation heat treatment with microwave heating method. The laboratory works involved are porosity and permeability measurement, Nuclear Magnetic Resonance, Xray CT scanning and Scanning Electron Microscopy imaging. In addition, numerical modelling concerning the heating reservoir with microwave has been conducted. Based on these data, the effects of microwave heating on tight sandstone samples were investigated.

Tight sandstone samples were heated with microwave. The maximum samples' surface temperature and heating time are $359.3^{\circ} \mathrm{C}$ in 10 minutes for ERREG-2, 504.1 $1^{\circ} \mathrm{C}$ in 5 minutes for WR-1, 337 $\mathrm{C}$ in 8 minutes for WR-11 and 483.7C for WR-14, time?. The internal temperature, which is believed to be higher than the surface temperature, was not accessible when we used microwave heating. However, in terms of temperature, it is not the higher the better, because some minerals may melt when the temperature is too high and block the other pore permanently. The temperature distribution in sandstone is determined by many factors, such as porosity, water saturation, salinity and mineralogy. In this paper, instead of conducting quantitative study on how these factors affect microwave heating, we put an emphasis on the effects of high temperature, generated by the interaction of microwave and sandstone, on the petrophysical properties. However, the quantitative study on factors affecting microwave heating of tight sandstones is of great significance in developing the application of microwave heating in FHT, it is necessary to conduct such investigation before the field application.

The most important issues we concerned during the gas production are reservoir quality and pore size distribution. The formation damages, such as water blocking and clay swelling, bring negative effects to the reservoir by blocking the flow path and reducing the relative permeability. In contrast, the microwave heating is able to recover the reservoir quality by evaporating water phase or even further improve the reservoir quality by creating micro-fractures and changing the mineralogy of sandstone. In our research, three different experiments, helium porosity and permeability, NMR, and X-ray CT numbers provide the relevant information and support each other. The helium porosity and permeability showed the improvement in the most direct way. Calibrated with helium porosity, NMR cumulative porosity confirms the increase of porosity, while the NMR T2 distribution indicates the changes of pore sizes within the sample: smaller pores diminished or developed into larger pores, so the amplitude of short NMR T2 decreased; micro-fractures generated between grains or in grains, so the amplitude of long NMR T2 increased. Deeper reasons were revealed by X-ray CT scanning. The X-ray CT images firstly provide the visualization of fractures (dimensions and positions) in the heated sample. The CT number, which can be obtained from stacked images, indicates the profile of porosity variations along the length of each sample. Sample WR-1, and WR-14 show fractures at one end of core plug in Figure 6 and indicate the increase of porosity in the corresponding position in the CT number profiles. In comparison, the sample ERREG-2 has a homogeneous porosity improvement, which may be because of the relative even temperature increase in this sample. The distributions of the porosity improvement are found heterogeneous in the other three samples. Actually, there are many reasons leading to a nonuniform temperature distribution:

1. The magnetic and electric field distributions are not even in the sandstones, so the intensities of different parts in the sample could vary from one to another.

2. The dielectric properties of grains are different due to mineral types, water content and porosity. Based on Equation 5, the relative permittivity determines the efficiency of microwave heating. However, the heterogeneous heating effects brought about the differential thermal expansion and leaded to the generation of micro-fractures.

3. The texture and structure of the tight sandstones affects the temperatures distribution, because they are related to the distribution of minerals in the sample. For the aggregation of minerals with high dielectric loss, it is more easily to be heated, so its temperature is high; for the aggregation of low loss minerals, its temperature is low. 
The penetration depth of heat treatment in the reservoir simulation reached to $0.4-0.5 \mathrm{~m}$, and the water phase can be completely removed within a radius of $0.25 \mathrm{~m}$. In the field application, the design of microwave heating devices is another important factor affecting the heating effectiveness and efficiency. By optimizing the microwave device, more energy can be delivered to the reservoir, and the treatment becomes more efficient and economical.

\section{Conclusions}

In summary, based on our preliminary study of microwave heating effects on tight sandstones, we come to the following conclusions:

1. By subjecting tight sandstone samples to microwave, the surface temperature of sandstone can be raised to around $400{ }^{\circ} \mathrm{C}$ or more (depends on the dielectric properties of sample), while the internal temperature is usually higher than that.

2. The shrinkage of clay minerals due to loss of water at high temperature has been noticed in this study. The differential thermal expansion and dehydration of clay minerals created fractures, so the porosity improved compared with original porosity. More importantly, the enhancement of permeability is higher than porosity for all studied samples, which has a great economical potential.

3. The micro-fractures grew into larger fractures or pores when sandstones were heated. This can be reflected by the change of T2 distribution of fully saturated samples: the area under short components of $\mathrm{T} 2$ curve decreased while long T2 increased. The analysis of SEM images (widely presented intergranular fractures after heating) and X-ray CT scanning images (obvious decrease of CT numbers after heating and presence of fractures) illustrate NMR results well.

4. Some minerals were changed due to microwave heating. By using the technique of XRD, calcite and feldspar were found disappeared after heating. Since they are common cement minerals in sandstone, the reservoir quality can benefit from the decomposition of these minerals.

5. The simulated depth of phase invasion was around 0.7 meter and the temperature reached up to $900^{\circ} \mathrm{C}$ within 1 day. Water phase has been completely removed within $25 \mathrm{~cm}$, although the water saturation reduced slightly in the depth beyond that. It is promising to bring down the water saturation with microwave heating in the near wellbore region.

6. The optimization of microwave heating in field may be achieved by improving the design of downhole microwave heating device. It is necessary to conduct further research on downhole device design in order to increase the depth of penetration and efficiency of energy conversion.

\section{Nomenclature}

$\nabla$

$E$

$B$
Nabla symbol ( $\nabla \cdot$ is divergence operator, $\nabla \times$ is a curl operator)

Electric field

Magnetic field

Electric charge density

Permittivity of free space $(8.854187817 \times 10-12 \mathrm{~F} / \mathrm{m})$

Permeability of free space $(1.2566370614 \times 10-6 \mathrm{H} / \mathrm{m})$

Current density

Absorbed power per unit volume

Frequency

Penetration Depth 
$\lambda_{0} \quad$ Wavelength

\section{Acknowledgments}

The authors of this manuscript would like to thank China Scholarship Council (CSC), The AustraliaChina Natural Gas Technology Partnership Fund and Unconventional Gas Research Group of Curtin University for financial and technical support. Appreciation to Dr. Lionel Esteban in CSIRO for providing X-ray CT scanning and technical support, to Ms. Elaine Miller, John de Laeter Centre of Curtin University for providing high quality SEM imaging, to Dr. Nguyen Tran for providing advice on microwave heating, to Ian Penny in CSIRO for preparing required aluminum tubes. Finally, thanks to the ANSYS and CMG for providing Academic License and ImageJ for providing free but powerful software.

\section{References}

Abernethy, E. R., 1976. Production increase of heavy oils by electromagnetic heating. J CAN PETROL TECHNOL, 15(03).

Anders, M. H., Laubach, S. E., and Scholz, C. H., 2014. Microfractures: A review. J STRUCT GEOL, 69, 377-394.

Atomic Energy of Canada Limited Research Company and Voss Associates Engineering Ltd., 1990. Microwaves and Minerals. Industrial Mineral Background Paper\#14.

Carrizales, M. A., Lake, L. W., \& Johns, R. T., 2010, January. Multiphase fluid flow simulation of heavy oil recovery by electromagnetic heating. In SPE Improved Oil Recovery Symposium. Society of Petroleum Engineers.

Carrizales, M.A., 2010. Recovery of stranded heavy oil by electromagnetic heating. Thesis.

Carroll, D., 1970. Clay minerals: a guide to their X-ray identification (Vol. 126). Geological society of America.

Clark, D.E. and Sutton, W.H., 1996. Microwave processing of materials. ANNU REV MATER SCI, 26(1), pp.299-331.

El-Dairy, M., Perez, J.M. and Poston, J.M., 1993. Petrophysical analysis of sandstones using CT scanning and conventional methods. AAPG BULL.

George R. Coates, L. Xiao and Manfred G. Prammer., 1999. NMR Logging Principles and Applications: Halliburton Energy Services.

Grim, R.E. and Bradley, W.F., 1940. Investigation of the Effect of Heat on the Clay Minerals Illite and Montmorillonite*. J AM CERAM SOC, 23(8), pp.242-248.

Hajpál, M. and Török, A., 2004. Mineralogical and colour changes of quartz sandstones by heat. ENVIRON GEOL, 46(3-4), pp.311-322.

Hoekstra, P., 1976. Rock, Frozen Soil and Ice Breakage by High Frequency Electromagnetic Radiation: A Review (No. CRREL-76-36). Cold Regions Research and Engineering Lab Hanover Nh. 
Holditch, S.A., 2006. Tight gas sands. J PETROL TECHNOL, 58(06), pp.86-93.

Jamaluddin, A.K.M., Hamelin, M., Harke, K., McCaskill, H. and Mehta, S.A., 1999. Field testing of the formation heat treatment process. J CAN PETROL TECHNOL, 38(03).

Jamaluddin, A.K.M., Vandamme, L.M., Nazarko, T.W. and Bennion, D.B., 1998. Heat treatment for clay-related near wellbore formation damage. J CAN PETROL TECHNOL, 37(01).

Jamaluddin, A.K.M., Vandamme, M. and Mann, B.K., 1995, January. Formation heat treatment (FHT): a state-of-the-art technology for near-wellbore formation damage treatment. In Annual Technical Meeting. Petroleum Society of Canada.

Kranz, R.L., 1983. Microcracks in rocks: a review. TECTONOPHYSICS, 100(1), pp.449-480.

Li, G., Meng, Y. and Tang, H., 2006. Clean up water blocking in gas reservoirs by microwave heating: laboratory studies. In International Oil \& Gas Conference and Exhibition in China. Society of Petroleum Engineers.

Liu, M.Y. and Zhao, G., 2012. Parametric study of heavy oil recovery by electromagnetic heating. COMSOL Conference 2012, Boston.

McGill, S.L., Walkiewicz, J.W. and Clark, A.E., 1995. Microwave Heating of Chemicals and Minerals. US Department of the Interior, Bureau of Mines.

Miesch, E.P. and Albright, J.C., 1967, January. A Study of Invasion Diameter. In SPWLA 8th Annual Logging Symposium. Society of Petrophysicists and Well-Log Analysts.

Mubiayi, M.P., 2013. Characterisation of sandstones: mineralogy and physical properties. Proceedings of the World Congress on Engineering.

Perez, J.M., 1993. Petrophysical Analysis of Sandstones Using CT Scanning and Conventional Methods.

Rider, M.H., 1986. The geological interpretation of well logs. Rider-French Consulting Ltd.

Sanmiguel, J.E., Mallory, D.G., Mehta, S.A. and Moore, R.G., 2001. Formation heat treatment process by combustion of gases around the wellbore. In Canadian International Petroleum Conference.

Petroleum Society of Canada.

Vermeulen, F.E. and Chute, F.S., 1983. Electromagnetic techniques in the in-situ recovery of heavy oils. J MICROWAVE POWER. 18(1), 15-29.

Wacker, B., Karmeileopardus, D., Trautmann, B., Helget, A. and Torlak, M., 2011. Electromagnetic Heating for In-situ Production of heavy oil and bitumen Reservoirs. In Canadian Unconventional Resources Conference. Society of Petroleum Engineers.

Wang, H., Rezaee, R. and Saeedi, A., 2015. Evaluation of Microwave Heating on Fluid Invasion and Phase Trapping in Tight Gas Reservoirs. In SPE Asia Pacific Unconventional Resources Conference and Exhibition. Society of Petroleum Engineers.

Wellington, S.L. and Vinegar, H.J., 1987. X-ray computerized tomography. J PETROL TECHNOL, 39(08). 885-898. 
Welton, J.E., 1984. SEM petrology atlas. Tulsa Oklahoma: American Association of Petroleum Geologists.

Wu, G., Wang, Y., Swift, G. and Chen, J., 2011. Physico-mechanical and thermo-mechanical characterisation of sandstone. Artificial Neural Networks in Biological and Environmental Analysis, pp.386.

Xie, H.P et, al., 1989. Analysis of rock fracture micro-mechanism (In Chinese). J China Coal Soc, 2 , pp.57-67.

Yao, Y., Liu, D., Che, Y., Tang, D., Tang, S., and Huang, W., 2009. Non-destructive characterization of coal samples from China using microfocus X-ray computed tomography. INT J COAL GEOL, 80(2), pp.113-123.

Zuo, J., Xie, H., Zhou, H., \& Peng, S., 2007. Thermal-mechanical coupled effect on fracture mechanism and plastic characteristics of sandstone. Science in China Series E: Technological Sciences, 50(6), pp.833-843. 